\title{
Association between visceral adiposity index, hirsutism and cardio- metabolic risk factors in women with polycystic ovarian syndrome: A cross-sectional study
}

\author{
Sanjeewani Fonseka ${ }^{1}$, Brabaharan Subhani, Chandrika N Wijeyaratne ${ }^{2}$, Indika B \\ Gawarammana $^{3}$, Nishan S Kalupahana ${ }^{4}$, Neelakanthi Ratnatunga ${ }^{5}$, Shanthini Rosairo ${ }^{6}$, \\ Kumarasiri P Vithane ${ }^{7}$
}

(Index words: adipocyte dysfunction, cardio-metabolic risk factors, hirsutism, polycystic ovarian disease, visceral adiposity index)

\begin{abstract}
Introduction Visceral adiposity index (VAI) is a mathematical index derived from the body mass index (BMI), waist circumference (WC), serum fasting triglyceride (TG) and high-density lipoprotein cholesterol (HDL-C). It reflects visceral adipocyte dysfunction (VAD) and is associated with cardiometabolic risk. Women with polycystic ovarian syndrome (PCOS) have adipocyte dysfunction, which is associated with metabolic disorders. Hirsutism in PCOS is considered to be due to high insulin levels which enhances androgen activity at the pilosebaceous unit.

Objectives To determine the association between VAI, hirsutism and cardiometabolic risk factors in patients with PCOS.

Methods A total of 99 patients aged 18-40 years with PCOS diagnosed by the Rotterdam consensus criteria2003 and a hirsutism score of 8 or more according to the Ferriman-Gallway Score (FGS) were studied. BMI, WC, fasting lipid profile, serum leptin, insulin, sex hormone binding globulin (SHBG), free-androgen index (FAI), fasting blood glucose (FBG) and oral glucose tolerance test (OGTT) were determined. Homeostasis model assessment (HOMA)-beta, HOMA-insulin resistance (IR) and VAI were calculated. Diameter and rate of hair growth at sideburns and chin; density of hair at sideburns were measured. Data were analyzed by SPSS-22.0.
\end{abstract}

Results There was no significant association between parameters of hirsutism and VAI. There was a significant association between VAI and OGTT, FAI, systolic and diastolic blood pressure: but not between VAI and other metabolic parameters.

Conclusion Visceral adipocyte dysfunction is closely linked to glucose intolerance and blood pressure in women with PCOS. However, hirsutism is unlikely to be due to adipocyte dysfunction.

Ceylon Medical Journal 2019; 64: 111-117

DOI: https://doi.org/10.4038/cmj.v64i3.8958

\section{Introduction}

\section{Background and rationale}

Visceral adipose tissue surrounds the intraabdominal organs. High visceral adipose tissue is associated with visceral adipocyte dysfunction which is characterized by an altered insulin sensitivity, lipolytic activity, a proinflammatory state and release of adipocytokines [1, 2]. They play a major role in cardiometabolic disease as it has been shown to be associated with diabetes, hypertension, lipid abnormalities and a high mortality rate [3]. Visceral

\begin{abstract}
${ }^{1}$ Department of Pharmacology, Faculty of Medicine, University of Peradeniya, ${ }^{2}$ Department of Obstetrics and Gynaecology, Faculty of Medicine, University of Colombo, ${ }^{3}$ Department of Medicine, Faculty of Medicine, University of Peradeniya, ${ }^{4}$ Department of Physiology, Faculty of Medicine, University of Peradeniya, ${ }^{5}$ Department of Pathology, Faculty of Medicine, University of Peradeniya, ${ }^{6}$ Department of Radiology, Faculty of Medicine, University of Peradeniya, ${ }^{7}$ Department of Community Medicine, Faculty of Medicine, University of Peradeniya, Sri Lanka.
\end{abstract}

Correspondence: SF, e-mail: <sanjeewani.fonseka@yahoo.com>. Received 9 July 2019 and revised version 1 September 2019 accepted 24 September 2019.

This is an open-access article distributed under the terms of the Creative Commons Attribution License, which permits unrestricted use, distribution, and reproduction in any medium, provided the original author and source are credited. 
adipocyte dysfunction leads to a rise in adipocytokines such as leptin, adiponectin, tumor necrosis factor-alpha and resistin [4]. This provides an objective index of the extent of adipose tissue dysfunction [5]. This index further predicts the conversion of metabolically healthy obesity to metabolically unhealthy obesity [6]. The quantitative analysis of visceral and cutaneous fat is carried out via MRI (Magnetic Resonance Imaging) or CT (Computed Tomography) and remain the gold standard for assessment of visceral adipose tissue though rather expensive and not routinely used in clinical practice [7].

The gender specific empirical mathematical model visceral adipose tissue index (VAI) is a surrogate marker of adipose tissue function [8]. It is estimated by the use of simple anthropometric (body mass index and waist circumference) and biochemical (triglycerides and high-density lipoprotein cholesterol) parameters [7].

The equation for VAI for females is as follows

$$
V A I=\left(\frac{\text { Waist Circumference }}{36.58+1.89 \times B M I}\right) \times\left(\frac{\text { Triglcerides }}{0.81}\right) \times\left(\frac{1.52}{\text { High Density Lipid-Cholesterol }}\right)
$$

The measurement of this value predicts the cardiometabolic risk of an individual early as it is indicative of insulin resistance and it is positively correlated with CT assessment of adipose tissue, the visceral to subcutaneous fat ratio and peripheral glucose utilization. It is a convenient index that can be easily used to assess the extent of adipocyte dysfunction in patients. Based on the above, age and gender specific quartiles have been calculated in order to classify the metabolic risk in these patients [7].

Polycystic ovary syndrome (PCOS) is a heterogeneous disorder characterized by a diverse collection of reproductive and metabolic abnormalities and part of the clinical spectrum of metabolic syndrome. It is characterized by hyperandrogenemia and chronic anovulation. In patients with PCOS VAI increases in relation to the severity of anovulation, insulin resistance and inflammation [4]. It predicts the cardio-metabolic risk of oligomenorrheic phenotype of PCOS. It enables to distinguish between metabolically healthy and metabolically unhealthy $\operatorname{PCOS}(9)$

Cardiometabolic risk is the overall risk of developing type 2 diabetes and cardiovascular diseases. The key risk factors which are known to be associated with enhanced cardio-metabolic risk include increased waist girth, elevated blood pressure, low HDL cholesterol, high triglycerides and impaired fasting glucose [10]. Abdominal visceral fat is a better determinant of cardiometabolic risk factors such as hypertension, type 2 diabetes, and dyslipidaemia than abdominal subcutaneous fat. Visceral adipocyte dysfunction is associated with a high cardiometabolic risk [7].

Hirsutism is the excessive growth of terminal hair in androgen dependent areas in women. It is a common feature seen in PCOS and is part of the diagnostic criteria; further, it gives rise to considerable anguish to women. It is caused by ovarian derived androgen excess and individual sensitivity of the pilosebaceous unit to androgen which supposedly mediated by high insulin levels [10].
Most patients with high insulin levels tend to have hirsutism as has been found to be associated with insulin resistance [11].

Most of the data available is for Caucasian patients aged 18 to 83 years and patients of South East Asian descent [7, 12]. Hence a lack of data for South Asians means caution needs to be exercised, when extrapolating these data to other ethnic groups.

Since hyperinsulinemia levels are attributed to be a causative mechanism for high androgenic activity at the pilosebaceous unit we postulated a probable correlation between the VAI and hirsutism in patients with PCOS and carried out this study to find, whether there is an association between VAI and hirsutism as well as cardiometabolic risk factors.

\section{Objectives}

\section{Method}

This is the baseline data from a clinical trial to assess the "effectiveness of ethinyl estradiol /cyproterone acetate and ethinyl estradiol/desogestrel with or without low-dose metformin on patients with polycystic ovary syndrome: A randomised, double-blind, placebocontrolled study". Data was collected from 99 female patients diagnosed to have PCOS. The inclusion criteria included females aged 18 to 40 years, diagnosed to have PCOS according to the Rotterdam consensus criteria and a hirsutism score of 8 or more according to the modified Ferriman-Gallway score. The patients who did not consent were excluded from the study. Patients with secondary causes of hirsutism such as idiopathic hirsutism, Cushing's syndrome, hyper-thecosis, androgen secreting tumor etc.

The study was conducted at the Pharmacology Department, Faculty of Medicine, University of Peradeniya. The patients were recruited from the Gynecology and Obstetrics Unit of Teaching Hospital Peradeniya and Teaching Hospital, Kandy. 
The basic demographic data such as name, age, marital status was collected from the patient. The weight, height, waist circumference, hip circumference, systolic and diastolic blood pressure was measured by a standard technique. The BMI (Body Mass Index) was calculated in these patients by the equation weight (in $\mathrm{kg}$ )/ height (in $\mathrm{m})^{2}$.

Venous blood samples were drawn from the patients after a 10-hour fasting duration. The Triglyceride (TG) and High-Density Lipid-Cholesterol (HDL-C) value were obtained from the lipid profile.

Further biochemical parameters such as leptin, sex hormone binding globulin, insulin, fasting blood glucose, oral glucose tolerance test (OGTT) and testosterone levels were measured and the free androgen index, homeostasis model assessment of insulin resistance (HOMA-IR), homeostasis model assessment of $\beta$ cell function (HOMA- $\beta$ ) and the area under the curve (AUC) for OGTT were derived.

The BMI, waist circumference, TG and HDL-C were used to calculate the gender specific-VAI and categorized according to the degree of visceral adipocyte dysfunction.

Hirsutism was assessed by determining the modified Ferriman-Gallway score, hair diameter and hair growth rate in chin and sideburns and hair density in sideburns. Hair density was measured by marking a $2 \mathrm{~cm} \times 2 \mathrm{~cm}$ square in front of the tragus and the number of hairs was counted using a digital photograph and Microsoft Paint software. Diameter of the hair was assessed by taking the average diameter of 5 strands of hair plucked measured from each site using Olympus B53 microscope (Olympus, Hamburg, Germany) and the software CellSense Entry (Olympus,
Japan). The rate of hair growth was assessed by taking the average length of 5 hair strands plucked from each site and measured by Vernier calipers and recording the last date of shaving [13].

Baseline characteristics were presented as mean \pm Standard Deviation (SD) for continuous variables; rates and proportions were calculated for categorical data. Normality of distribution for quantitative data was assessed by the Kolmogorov-Smirnov test. The variables that did not show a normal distribution were logtransformed. The categorical data was analyzed by the Man-Whitney U test.

\section{Results}

\section{Descriptive Statistics}

A total of 99 subjects were included in the final analysis of the baseline data of patients who were part of the clinical trial. The VAI value was analyzed by quartiles to examine the relationship of the clinical and metabolic characteristics according to the VAI values. Anthropometric and clinical variables, biochemical variables and parameters of hirsutism according to VAI quartiles are stated in Table 1, 2 and 3 respectively.

Correlation between natural logarithms of clinical, biochemical variables and natural logarithms are shown in Table 4. Correlation between the natural logarithms of VAI and natural logarithms of parameters of hirsutism revealed hair growth on the left side $r=-0.047(0.646)$, right side $r=-0.012(0.904)$ and chin of $-0.044(0.664)$; density of hair growth $r=-0.099(0.33)$; diameter on the left side $r=0.015(0.886)$, right side $r=0.079(0.438)$ and chin $r=0.145(0.145)$ and FGS $r=0.019(0.854)$.

Table 1. Anthropometric and clinical variables according to VAl quartiles Mean (SD)

\begin{tabular}{|c|c|c|c|c|}
\hline & Normal & Mild & Moderate & Severe \\
\hline Number & 62 & 6 & 3 & 27 \\
\hline Age & $23.89(5.53)$ & $26.67(6.74)$ & $30.0(8.66)$ & $26.48(8.11)$ \\
\hline Height & $155.92(4.9)$ & $150.58(18.22)$ & $155.5(11.63)$ & $154.0(6.7)$ \\
\hline Weight & $64.74(15.2)$ & $71.28(11.9)$ & $63.1(11.56)$ & $70.14(10.3)$ \\
\hline Waist Circumference & $80.93(15.58)$ & $88.5(6.59)$ & $94.67(14.6)$ & $90.9(9.09)$ \\
\hline Waist/Hip Ratio & $0.803(0.094)$ & $0.862(0.035)$ & $0.86(0.047)$ & $0.88(0.049)$ \\
\hline BMI & $26.7(5.75)$ & $28.22(3.42)$ & $25.89(1.034)$ & $29.13(3.72)$ \\
\hline Systolic Blood Pressure & $115.56(13.55)$ & $118.67(9.2)$ & $118.67(9.6)$ & $124.7(12.8)$ \\
\hline Diastolic Blood Pressure & $76(10.2)$ & $75(11.1)$ & $81(11.5)$ & $84.22(8.3)$ \\
\hline
\end{tabular}


Table 2. Biochemical variables according to VAl quartiles Mean (SD)

\begin{tabular}{|c|c|c|c|c|}
\hline & Normal & Mild & Moderate & Severe \\
\hline Number & 62 & 6 & 3 & 27 \\
\hline Total Cholesterol (TC) & $184.93(41.44)$ & $217.5(29.14)$ & $181.33(41.36)$ & $202.26(28.56)$ \\
\hline Triglyceride & $92.03(25.15)$ & $118.8(23.8)$ & $143.67(29.5)$ & $204.56(78.1)$ \\
\hline LDL $\quad 120.67(35.18)$ & $154.4(32.4)$ & $110.9(45.6)$ & $124.4(21.8)$ & \\
\hline HDL $\quad 47.8(17.09)$ & $38.83(5.46)$ & $41.67(6.35)$ & $37.16(10.45)$ & \\
\hline $\mathrm{TC} / \mathrm{HDL}-\mathrm{C}$ ratio & $5.59(10.93)$ & $5.71(1.18)$ & $4.46(1.42)$ & $5.41(1.07)$ \\
\hline Leptin $23.4(24.1)$ & $19.18(17.9)$ & $35.1(14.7)$ & $26.15(17.1)$ & \\
\hline Testosterone & $8.6(51.0)$ & $4.67(3.78)$ & $5.37(3.95)$ & $2.79(2.33)$ \\
\hline Free Androgen Index & $4.57(5.7)$ & $9.36(14.9)$ & $1.66(1.56)$ & $5.25(4.2)$ \\
\hline SHBG $97.24(79.95)$ & $74.1(53.9)$ & $85.21(94.9)$ & $78.48(61.6)$ & \\
\hline Insulin $8.01(12.8)$ & $12.82(22.8)$ & $2.9(2.95)$ & $6.32(10.71)$ & $6.4(11.14)$ \\
\hline HOMA-B & $105.9(314.6)$ & $32.9(56.5)$ & $17.66(28.9)$ & $154.19(413.4)$ \\
\hline FBS $\quad 83.9(19.5)$ & $80.17(8.52)$ & $69.66(9.5)$ & $87.15(18.1)$ & \\
\hline OGTT (2-hour value) & $106.3(19.3)$ & $111.67(25.1)$ & $112.33(24.1)$ & $117.15(24.5)$ \\
\hline OGTT(AUC) & $212.5(33.1)$ & $202.3(30.3)$ & $212.0(48.9)$ & $235.3(81.4)$ \\
\hline
\end{tabular}

Table 3. Parameters of hirsutism according to VAl quartiles Mean (SD)

\begin{tabular}{|c|c|c|c|c|}
\hline & Normal & Mild & Moderate & Severe \\
\hline Number & 62 & 6 & 3 & 27 \\
\hline Hair Growth Rate-Left sideburns & $1.43(0.84)$ & $1.61(0.97)$ & $1.14(0.55)$ & $1.28(0.55)$ \\
\hline Hair Growth Rate-Right sideburns & $1.32(0.57)$ & $1.25(0.67)$ & $1.17(0.72)$ & $1.37(0.6)$ \\
\hline Hair Growth Rate-Chin & $0.29(0.17)$ & $0.32(0.19)$ & $0.23(0.11)$ & $0.26(0.11)$ \\
\hline Density of Hair Growth & $100.23(61.7)$ & $73.44(43.8)$ & $69.33(44.5)$ & $106.76(67.56)$ \\
\hline Diameter of Hair-Left sideburns & $65.37(29.44)$ & $72.53(22.24)$ & $95.7(34.4)$ & $66.39(22.86)$ \\
\hline Diameter of Hair-Right sideburns & $63.41(24.4)$ & $77.89(21.5)$ & $76.57(10.9)$ & $69.89(22.86)$ \\
\hline Hair Growth Rate-Chin & $70.19(31.3)$ & $84.5(30.7)$ & $90.98(26.2)$ & $78.61(26.86)$ \\
\hline Total FGS & $19.44(5.77)$ & $24.0(5.8)$ & $20.0(6.00)$ & $18.89(5.8)$ \\
\hline
\end{tabular}

Table 4. Correlation between natural logarithms of clinical, biochemical variables and natural logarithms of VAI

\begin{tabular}{ll}
\hline & $\begin{array}{c}\text { VAI correlation } \\
(p \text { value })\end{array}$ \\
\hline Leptin & $0.166(0.104)$ \\
Testosterone & $0.123(0.22)$ \\
Free Androgen Index & $0.250(0.014)$ \\
SHBG & $-0.133(0.199)$ \\
Insulin & $-0.021(0.84)$ \\
HOMA-IR & $-0.101(0.338)$ \\
HOMA-B & $0.081(0.444)$ \\
FBS & $0.013(0.898)$ \\
OGTT (2-hour value) & $0.194(0.054)$ \\
OGTT (AUC) & $0.122(0.228)$ \\
Systolic Blood Pressure & $0.346(<0.05)$ \\
Diastolic Blood Pressure & $0.321(<0.05)$ \\
\hline
\end{tabular}


Correlation between the free androgen index and parameters of hirsutism revealed hair growth on the left side $r=0.021(p=0.841)$, right side $r=0.003(p=0.976)$ and chin of $0.022(\mathrm{p}=0.828)$; density of hair growth $\mathrm{r}=-0.195$ ( $\mathrm{p}=0.055)$; diameter on the left sider $=0.181(\mathrm{p}=0.077)$, right side $r=0.136(\mathrm{p}=0.183)$ and chin $\mathrm{r}=0.059(\mathrm{p}=0.569)$ and $\mathrm{FGSr}=0.060(\mathrm{p}=0.559)$.

Correlation between the testosterone and parameters of hirsutism revealed hair growth on the left side $r=-0.063$ $(p=0.536)$, right side $r=-0.108(p=0.286)$ and chin of -0.060 $(\mathrm{p}=0.560)$; density of hair growth $\mathrm{r}=-0.133(\mathrm{p}=0.190)$; diameter on the left side $\mathrm{r}=-0.067(\mathrm{p}=0.510)$, right side $\mathrm{r}=-0.059(\mathrm{p}=0.559)$ and chin $\mathrm{r}=-0.066(\mathrm{p}=0.517)$ and FGS $r=-0.199(\mathrm{p}<0.05)$.

Correlation between the insulin and parameters of hirsutism revealed hair growth on the left side $r=-0.062$ $(p=0.544)$, right side $r=-0.119(p=0.242)$ and chin of -0.067 $(\mathrm{p}=0.512)$; density of hair growth $\mathrm{r}=-0.071(\mathrm{p}=0.484)$; diameter on the left side $\mathrm{r}=0.132(\mathrm{p}=0.193)$, right side $r=0.030(p=0.768)$ and chin $r=0.092(p=0.363)$ and FGSr $=0.038(\mathrm{p}=0.708)$.

Correlation between the SHBG and parameters of hirsutism revealed hair growth on the left side $r=0.035$ (0.734), right side $r=0.060(0.560)$ and chin of $0.033(0.747)$; density of hair growth $r=-0.104(0.308)$; diameter on the left side $r=-0.128(0.210)$, right side $r=-0.126(0.210)$ and chin $r=-0.060(0.56)$ and FGS $r=-0.010(0.921)$.

Adiposity index demonstrates a skewed distribution with a kurtosis of 6.274. The patients were divided into two categories based on the VAI as metabolically unhealthy and healthy (Table 5). The VAI cut-off value for severe visceral adipocyte dysfunction was used to differentiate between metabolically healthy and unhealthy. Since most variables showed a non-normal distribution, as well as the difference of the number of subjects between the two groups Mann-Whitney U test was carried out.

Table 5. Difference of the clinical, biochemical variables and parameters of hirsutism in between the two groups

\begin{tabular}{|c|c|c|c|c|}
\hline & $\begin{array}{c}\text { Metabolically } \\
\text { Healthy } \\
\text { (Mean Rank) }\end{array}$ & $\begin{array}{c}\text { Metabolically } \\
\text { Unhealthy } \\
\text { (Mean Rank) }\end{array}$ & Test statistic & $P$ value \\
\hline Weight & 47.05 & 57.04 & 759.5 & 0.095 \\
\hline Age & 46.69 & 58.83 & 733.5 & 0.06 \\
\hline Waist Circumference & 45.31 & 62.52 & 634 & 0.008 \\
\hline Waist-Hip Ratio & 42.62 & 69.69 & 440.5 & $<0.05$ \\
\hline BMI & 46.4 & 59.59 & 713 & 0.042 \\
\hline Systolic Blood Pressure & 44.67 & 64.2 & 588.5 & 0.003 \\
\hline Diastolic Blood Pressure & 43.49 & 67.35 & 503.5 & $<0.05$ \\
\hline Leptin & 46.37 & 56.17 & 736.5 & 0.129 \\
\hline SHBG & 49.98 & 48.24 & 924.5 & 0.787 \\
\hline Testosterone & 49.05 & 52.54 & 903.5 & 0.59 \\
\hline Androgen Index & 46.07 & 56.59 & 747 & 0.099 \\
\hline Insulin & 50.63 & 48.31 & 926.5 & 0.721 \\
\hline HOMA-IR & 50.16 & 47.76 & 911.5 & 0.709 \\
\hline HOMA-B & 47.39 & 55.64 & 809 & 0.234 \\
\hline FBS & 49.01 & 52.65 & 900.5 & 0.574 \\
\hline OGTT-2-hour value & 46.29 & 59.89 & 705 & $<0.05$ \\
\hline OGTT-AUC & 47.74 & 56.04 & 809 & 0.2 \\
\hline Triglyceride & 37.95 & 82.13 & 104.5 & $<0.05$ \\
\hline LDL & 49.59 & 51.09 & 942.5 & 0.817 \\
\hline HDL & 56.14 & 33.63 & 530 & 0.001 \\
\hline TC/HDL-C ratio & 42.69 & 69.8 & 445.5 & $<0.05$ \\
\hline Hair Growth Rate-Left sideburns & 50.37 & 49.02 & 945.5 & 0.835 \\
\hline Hair Growth Rate-Right sideburns & 49.54 & 51.22 & 939 & 0.795 \\
\hline Hair Growth Rate-Chin & 50.33 & 49.13 & 948.5 & 0.853 \\
\hline Density of Hair Growth & 49.31 & 51.83 & 922.5 & 0.697 \\
\hline Diameter of Hair-Left sideburns & 50.01 & 49.98 & 971.5 & 0.997 \\
\hline Diameter of Hair-Right sideburns & 48.89 & 52.96 & 892 & 0.53 \\
\hline Hair Growth Rate-Chin & 48.26 & 54.65 & 845.5 & 0.324 \\
\hline Ferriman-Gallway Score & 50.7 & 48.13 & 921.5 & 0.691 \\
\hline
\end{tabular}




\section{Discussion}

The main findings in this study are that about $30 \%$ of the patients have the metabolically-unhealthy variant of PCOD, there is no correlation between parameters of hirsutism and the visceral adiposity index and VAI is associated with an elevated cardio-metabolic risk.

Visceral adiposity index predicts transition from metabolically healthy to unhealthy status; hence almost $30 \%$ of our patients were metabolically unhealthy which places them at a higher risk of cardiovascular morbidity and mortality, hence it is prudent to follow up these patients to detect adverse cardiovascular outcomes in the future [6].

Majority of the patients were overweight, with an increased waist circumference, increased waist to hip ratio, impaired glucose tolerance and high systolic and diastolic blood pressure readings which places them in the category of metabolic syndrome which is a predictor of adverse cardiovascular outcome. They are at high risk of conversion to a state of metabolic ill health in the future even despite of normal visceral adipose dysfunction at the moment [1].

Although about $30 \%$ of our patients had severe VAD they did not have higher fasting insulin levels than the patients with normal VAD. Our findings do not support the hypothesis of hyperinsulinaemia as a contributory factor for hirsutism.

OGTT 2-hour value was higher among the metabolically unhealthy than the metabolically healthy patients but the AUC for OGTT did not demonstrate a significant difference between the two groups. Though very few patients had overt diabetes within our population there was evidence of glucose intolerance which correlated with the VAD.

There was an overall increase in the parameters on the lipid profile. Triglyceride and HDL-C were elevated in the metabolically unhealthy patients compared to metabolically healthy patients whereas the reverse was observed with regard to HDL levels. Triglyceride levels in particular have been associated with VAD and poor metabolic health.

The hair growth rate, hair diameter, hair density and modified FGS has not been previously studied in Sri Lankan individuals thus, it is difficult to make a comparison of the values observed in these women. There is lack of both normative data and hirsutism indices in our population [14]. Testosterone, insulin and free androgen index were elevated but there was no significant correlation between them and visceral adipocyte dysfunction or parameters of hirsutism [2].

SHBG, HOMA-IR, HOMA- $\beta$, insulin and leptin levels were elevated in our population compared to age stratified cut-off values but no difference was observed between metabolically healthy and unhealthy patients and do not appear to be correlated with the VAI which is both an index of VAD and insulin resistance [15]. Systolic and diastolic blood pressures significantly correlated with the VAI and this could possibly be related to endothelial dysfunction [16]. However, since this was a cross-sectional study the probability of a subsequent development of hypertension could not be predicted.

Since this was the baseline data obtained from a clinical trial the major limitation of the study was the inadequacy of the sample size which may be a reason for the lack of detection of associations which have been observed in prior studies.

\section{Conclusions}

Visceral adipocyte dysfunction (as measured by VAI) is closely linked to impaired glucose tolerance and blood pressure in women with PCOS. Hirsutism is unlikely to be related to adipocyte dysfunction in these women. A larger sample size may be required to discern the presence of an association between the above-mentioned variables in PCOS.

\section{Acknowledgments}

The technical staff members at the Departments of Pharmacology, Pathology, Biochemistry and Nuclear Medicine Unit.

\section{Availability of data}

The data of findings of this study is available from the corresponding author (SF) on request.

\section{Conflict of interests}

No conflicts of interest have been declared.

\section{References}

1. Bergman RN, Kim SP, Catalano KJ, et al. Why Visceral Fat is Bad: Mechanisms of the Metabolic Syndrome. Obesity 2006; 14(S2): 16S-9S.

2. De Simone M, Verrotti A, Iughetti L, et al. Increased visceral adipose tissue is associated with increased circulating insulin and decreased sex hormone binding globulin levels in massively obese adolescent girls. $J$ Endocrinol Invest 2001; 24(6): 438-44.

3. Bozorgmanesh M, Hadaegh F, Azizi F. Predictive performance of the visceral adiposity index for a visceral adiposity-related risk: type 2 diabetes. Lipids Health Dis 2011; 10: 88.

4. Al-Daghri NM, Al-Attas OS, Alokail MS, et al. Visceral adiposity index is highly associated with adiponectin values and glycaemic disturbances. Eur J Clin Invest 2013; 43(2): 183-9.

5. Amato MC, Giordano C, Galia M, et al. Visceral Adiposity Index: a reliable indicator of visceral fat function associated with cardiometabolic risk. Diabetes Care 2010; 33(4): 920-2. 
6. Kang YM, Jung CH. Visceral adiposity index predicts the conversion of metabolically healthy obesity to an unhealthy phenotype. 2017; 12(6): e0179635.

7. Amato MC, Giordano C, Pitrone M, Galluzzo A. Cut-off points of the visceral adiposity index (VAI) identifying a visceral adipose dysfunction associated with cardiometabolic risk in a Caucasian Sicilian population. Lipids Health Dis 2011; 10: 183.

8. Amato MC, Giordano C. Visceral Adiposity Index: An Indicator of Adipose Tissue Dysfunction. International Journal of Endocrinology 2014; 2014: 7.

9. Shuster A, Patlas M, Pinthus JH, Mourtzakis M. The clinical importance of visceral adiposity: a critical review of methods for visceral adipose tissue analysis. The British Journal of Radiology 2012; 85(1009): 1-10.

10. Wilson PWF, Meigs JB. Cardiometabolic risk: a Framingham perspective. International Journal of Obesity 2008; 32: S17.

11. Azziz R, Carmina E, Sawaya ME. Idiopathic hirsutism. Endocr Rev 2000; 21(4): 347-62.

12. Talaei A, Adgi Z, Mohamadi Kelishadi M. Idiopathic hirsutism and insulin resistance. International Journal of Endocrinology 2013.

13. Oh S-K, Cho AR, Kwon Y-J, Lee H-S, Lee J-W. Derivation and validation of a new visceral adiposity index for predicting visceral obesity and cardiometabolic risk in a Korean population. Plos One 2018; 13(9): e0203787.

14. Hayashi S, Miyamoto I, Takeda K. Measurement of hair growth by optical microscopy and image analysis; 1991.

15. Loussouarn G, Lozano I, Panhard S, Collaudin C, El Rawadi C, Genain G. Diversity in human hair growth, diameter, colour and shape. An in vivo study on young adults from 24 different ethnic groups observed in the five continents. Eur J Dermatol 2016; 26(2): 144-54.

16. Gayoso-Diz P, Otero-Gonzalez A, Rodriguez-Alvarez MX, et al. Insulin resistance (HOMA-IR) cut-off values and the metabolic syndrome in a general adult population: effect of gender and age: EPIRCE cross-sectional study. BMC Endocr Disord 2013; 13: 47.

17. Yang F, Wang G, Wang Z, et al. Visceral adiposity index may be a surrogate marker for the assessment of the effects of obesity on arterial stiffness. Plos One 2014; 9(8): e104365-e. 